\title{
ENSAYOS
}




\section{ESTRATEGIAS DE DESARROLLO Y EDUCACION: EL DESAFIO DE LA GESTION PÚBLICA}

Juan Carlos Tedesco*

La educación vuelve a ser considerada una prioridad en la agenda de discusiones sobre estrategias de desarrollo. Este hecho es particularmente visible en los países desarrollados, donde los problemas que afectan a los sistemas educativos y las alternativas para enfrentarlos han dejado de ocupar exclusivamente la atención de los especialistas para atraer la de los dirigentes políticos, los medios de comunicación de masas y, por supuesto, las familias.

¿Cuál es la novedad en este debate? Alguien podría decir, con razón, que la educación siempre fue considerada una prioridad tanto en las políticas públicas como en las estrategias familiares. Sin embargo, un rápido análisis histórico de las diferentes concepciones desde las cuales se interpretaron y - en cierta medida- se orientaron las decisiones sobre política educativa permitirá distinguir algunos cambios significativos.

Al respecto, existe consenso en distinguir al menos tres grandes paradigmas que se corresponden con tres períodos históricos diferentes en las relaciones entre educación y desarrollo social ${ }^{1}$.

En el primero de ellos, cuya vigencia alcanzó hasta comienzos del siglo $X X$, la educación fue concebida como una actividad sistemática, efectuada desde la escuela y orientada a formar a las personas en su condición de ciudadanos. El liberalismo de fines del siglo XIX y el proceso de consolidación de los estados nacionales enfatizaron una acción educativa destinada a incorporar a todos los individuos en marcos y códigos culturales comunes y ejercer sus derechos cívicos elementales.

Consolidado el proceso de formación de la ciudadanía y en el contexto de las exigencias de la reconstrucción de posguerra, el modelo liberal fue reemplazado por un paradigma que concebía la educación a partir de su contribución al aumento de productividad de la fuerza de trabajo. La metáfora de la formación del ciudadano fue reemplazada por la metáfora de los recursos humanos y las decisiones educativas fueron procesadas y evaluadas como decisiones de inversión de capital.

Una vez resueltas las demandas de la reconstrucción y en el contexto de una creciente rigidez del mercado de trabajo, la educación comenzó a ser considerada como un mero aparato reproductor ya sea de la estructura jerarquizada de la fuerza de trabajo, ya sea de la constelación de valores asociada a dicha jerarquía.

Actualmente, en cambio, el escepticismo acerca del papel de la educación en el crecimiento económico y en la vigencia de patrones políticos democráticos parece superado. En su lugar, asistimos al diseño y difusión de un enfoque (ya que parece prematuro hablar de un nuevo modelo o paradigma) que otorga a la educación (más específicamente, a la escuela) un papel significativo en la explicación de los logros de

\footnotetext{
* Director Oficina Regional de Unesco. OREALC. Santiago de Chile.

${ }^{1}$ Un análisis más detallado de este tema puede verse en Juan C. Tedesco. El desafío educativo, calidad y democracia. Buenos Aires, Grupo Editor Latinoamericano, 1989, cap. 1.
} 
aprendizaje de los alumnos y que enfatiza la necesidad de analizar el papel de la educación en el proceso de desarrollo desde una perspectiva más integral que en el pasado. Por un lado, este enfoque se apoya en la revalorización del modelo tradicional que ponía el acento en el rol político de la educación. La formación del ciudadano, tanto en los países que están tratando de consolidar procesos democráticos como en los de mayor nivel de desarrollo, donde los actores tienden a obtener crecientes grados de autonomía, constituye un factor desde el cual se generan fuertes demandas sobre la tarea educativa. Estas demandas incluyen desde las clásicas propuestas de formación en el respeto a los derechos humanos y la paz hasta las más actuales preocupaciones por rebrotes racistas, defensa de las minorías y la protección ambiental. Pero al mismo tiempo, este nuevo enfoque advierte que la necesidad de fortalecer las capacidades productivas de las personas ha adquirido actualmente una importancia crucial. El mundo del trabajo se ha complejizado en forma notoria y la actividad productiva se articula cada vez más estrechamente con la actividad intelectual: por un lado, la producción requiere mayores niveles de logro en determinadas capacidades humanas como la creatividad, la inteligencia, la selección de información; por el otro, la actividad productiva no solo consume conocimientos, sino que los produce. Por esta razón, actualmente, una educación desligada del mundo del trabajo no sólo es regresiva desde el punto de vista económico sino empobrecedora desde la perspectiva del desarrollo integral de la personalidad individual.

Las modificaciones en los paradigmas a partir de los cuales se analiza el papel de la educación también reflejan cambios en la concepción del propio proceso de desarrollo. En este sentido, la modificación más importante consiste en concebir los que ahora se denominan "factores humanos" y sus productos (información, creatividad, inteligencia, etc.), desde una perspectiva sistémica. En este sentido, las personas y sus capacidades comienzan a ser consideradas como el objetivo del desarrollo y como factor central de las estrategias para lograrlo.

Esta somera y muy esquemática visión del proceso seguido por el pensamiento educativo responde a la situación de los países capitalistas avanzados, particularmente a los europeos. En América Latina, en cambio, el debate educativo no ha alcanzado ni la prioridad ni la articulación que se aprecia en dichos países. Los modelos mencionados tuvieron una vigencia parcial y fueron reemplazados sin que los problemas a los cuales estaban respondiendo hubieran sido resueltos. La explicación de esta desarticulación ha sido objeto de abundante literatura. Sin embargo, las explicaciones tradicionales deben ser reevaluadas en función del nuevo contexto creado por la crisis y por las estrategias diseñadas para salir de ella.

En este sentido, existe concenso en reconocer que la crisis no puede ser analizada como un fenómeno exclusivamente externo, es decir, independiente de los rasgos propios del estilo de desarrollo vigente en la región antes de la crisis. La revisión de las características de dichos estilos, a la luz de los resultados de la crisis, permite apreciar que uno de los rasgos más notorios del pasado ha sido la significativa distancia que ha existido entre la capacidad expresiva - entendida como capacidad para formular propuestas y resolver retóricamente los problemas - y la capacidad para producir resultados. El foco de análisis de este artículo será, en consecuencia, el análisis de la situación educativa regional desde el punto de vista de los mecanismos de gestión asociados a los desafíos futuros. 


\section{El impacto de la crisis sobre la educación}

La crisis ha provocado, obviamente, un severo retroceso en los logros educativos de América Latina. Desde este punto de vista, es importante señalar que el impacto de la crisis no ha sido similar en todas las regiones ${ }^{2}$. Los datos sobre gasto público destinado a educación por habitante permiten apreciar, por ejemplo, que durante el período 19801986, tanto en los países desarrollados como en algunas regiones en desarrollo (Asia y Estados Árabes), se produjo un aumento de la inversión en educación. El retroceso afectó solamente a los países de África y de América Latina y el Caribe, donde las magnitudes se redujeron en forma significativa (ver cuadro 1 ).

Cuadro 1. Gasto público en educación por habitante (en US\$ a precios corrientes)

\begin{tabular}{lrrr}
\hline & 1980 & 1985 & 1986 \\
\hline Total mundial & 144 & 144 & 141 \\
Todos los países desarrollados & 471 & 508 & 595 \\
Países árabes & 113 & 138 & 141 \\
América Latina y Caribe & 88 & 63 & 60 \\
Asia (excepto países árabes) & 38 & 39 & 52 \\
Todos los países en desarrollo & 29 & 27 & 27 \\
África & 33 & 20 & 15 \\
\hline
\end{tabular}

Fuente: UNESCO, Statiscal Yearbook, 1988.

La disminución del gasto público en educación está acompañada, además, por una sensible disminución de la capacidad de las familias tanto para asumir privadamente los costos de la educación como para garantizar condiciones materiales de vida a sus hijos que permitan aprovechar la oferta educacional existente. Como resultado de este conjunto de factores podríamos identificar cinco fenómenos como los principales efectos de la crisis en el sector educativo:

i) En primer lugar, el incremento de los índices de repetición y fracaso escolar, especialmente en la escuela básica. De acuerdo a estimaciones de la Unesco basadas en informes oficiales, entre 1975 y 1983 el número de repitentes en las escuelas primarias de América Latina ascendió de 5.7 millones a 8.5 millones de niños. Pero estudios recientes realizados con metodologías más complejas, señalan que estas cifras estarian subestimando significativamente la magnitud del problema ${ }^{3}$. No es éste el lugar ni el momento para un análisis detallado de los indicadores de la repetición escolar. Baste decir, sin embargo, que América Latina constituye hoy en día la región en el mundo con mayores tasas de repetición escolar y que este fenómeno se concentra desde el punto de vista social —en los sectores pobres, rurales y marginales urbanos y desde el punto de

\footnotetext{
${ }^{2}$ Para una evaluación del impacto de la crisis a nivel internacional, véase Jacques Hallak. Investing in the Future; setting educational priorities in the developing world. Unesco/liep/Pegamon Press, 1990. Para el caso de América Latina, Fernando Reimers. Deuda externa y financiamiento de la educación; su impacto en Latinoamérica. Orealc/Unesco, Santiago de Chile, 1990.

${ }^{3}$ Véase Ernesto Schifelbein. "Repetición: la última barrera para universalizar la educación primaria en América Latina”, en OREALC/UNESCO. Boletín del Proyecto Principal de Educación en América Latina y el Caribe, No. 18, Santiago, Chile, abril de 1989.
} 
vista pedagógico, en los primeros grados de la escuela básica, es decir, directamente vinculado al aprendizaje de la lectura y la escritura.

La magnitud de este fenómeno permite sostener que no se trata de un problema exclusivamente pedagógico, sino que es el reflejo de una acumulación de carencias materiales, culturales y-por supuesto- educativas ${ }^{4}$. Pero el punto sobre el cual corresponde enfatizar en el contexto de nuestras discusiones es el que se refiere al bajo nivel de responsabilidad por los resultados con los cuales operan los sistemas educativos. Tasas tan altas de fracaso no suelen desencadenar respuestas orientadas a la solución del problema sino que — frecuentemente — la responsabilidad del fracaso es atribuida a la propia víctima del proceso.

u) En segundo lugar, los datos disponibles muestran que se ha producido una interrupción del proceso de incorporación de los hijos de familias de sectores populares a los niveles posbásicos del sistema educativo. Desde este punto de vista, los datos estadísticos muestran una visible disminución en el ritmo de expansión de la enseñanza media y superior. Desde 1980 en adelante, las tasas de crecimiento de los niveles medio y superior son cada vez más bajas en un número creciente de países. Con respecto a la enseñanza media, los datos referidos a América Latina indican un descenso en las tasas de crecimiento de la matrícula de $7.3 \%$ en el período $1960-1980$ al $3.8 \%$ en el período 1980-1986. Para la enseñanza superior, en el mismo período, el descenso fue de una tasa de $10.4 \%$ a una tasa de $5.6 \%$. Estas cifras demuestran, por un lado, que se agotó el proceso de expansión "fácil" del sistema educativo vigente entre 1950 y 1980 y, por el otro, que para permitir el acceso de nuevos sectores sociales a la enseñanza media y superior serán necesarios cambios profundos en las condiciones de vida -empleo e ingresos- de dichos sectores.

iii) En tercer lugar, la crisis está provocando el aumento de la diferenciación interna del sistema educativo. Como se sabe, el deterioro en las condiciones materiales de vida afectó no sólo a los sectores que vivían en condiciones de pobreza antes de la crisis, sino también a importantes grupos poblacionales que en el pasado habían logrado acceso al consumo de ciertos bienes y servicios. Con respecto a la educación, este fenómeno se reflejó tanto en el aumento de la matrícula de los niveles medio y superior como en la expansión de la enseñanza privada. En la última década, y a pesar de las fuertes orientaciones privatizadoras, muchos países mostraron tendencias al incremento de la matrícula escolar pública. Dichos aumentos fueron más significativos en el nivel preescolar y en los niveles medio y superior y reflejan el comportamiento de capas medias que han perdido capacidad para asumir privadamente los costos de la educación. En un contexto de fuerte reducción del gasto público en educación, el aumento de la matrícula del sector público está asociado a una mayor diferenciación interna en el sistema educativo, donde el sector público adopta características cada vez mas masivas y el sector privado características cada vez más elitistas.

Los puntajes en pruebas estandarizadas de rendimiento en la educación primaria, en la media docena de países que las utilizan, indican, por ejemplo, que los promedios de rendimiento en las escuelas que atienden a alumnos de niveles socio-económicos bajos son equivalentes a la mitad, o a la tercera parte de los puntajes de las escuelas que atienden algunos de los niveles socioeconómicos altos.

\footnotetext{
${ }^{4}$ Véase Juan C. Tedesco. "Modelo pedagógico y fracaso escolar", en Revista de la Cepal, No. 21, Santiago, Chile, diciembre de 1983.
} 
iv) En cuarto lugar y estrechamente asociado al punto anterior, la crisis está asociada al deterioro de la calidad de la educación. El concepto de calidad de la educación es ambiguo y complejo. Sin embargo, es evidente que más allá de cualquier discusión teórica sobre el tema de la calidad y sobre las metodologías de medición de resultados, hay dos datos que avalan la hipótesis del impacto de la crisis sobre esta variable: el salario docente y la proporción del presupuesto educativo destinado a equipamiento, capacitación, infraestructura, libros de texto, etc.

La reducción del poder adquisitivo del salario docente es un fenómeno generalizado en América Latina. Sus efectos son múltiples y bien conocidos: el abandono de la profesión por parte de los docentes más calificados, el ausentismo y la desmoralización creciente del personal de la educación, huelgas prolongadas, etc. Entre otras consecuencias, estos fenómenos han provocado una sensible disminución en el tiempo de exposición al aprendizaje al cual tienen acceso los alumnos, que suele ser muy inferior al formalmente establecido en los calendarios escolares. Por otra parte, también es sabido que prácticamente en todos los países de la región el porcentaje del presupuesto destinado a salarios alcanza magnitudes cercanas al $90 \%$, dificultando cualquier tentativa de mejorar la calidad a través de inversiones en infraestructura, en equipos, en perfeccionamiento del personal y en innovaciones educativas. Esta situación ya era particularmente deficitaria antes de la crisis. Un estudio efectuado sobre 66 países con datos cercanos a 1980 mostró que América Latina gastaba casi 9 US\$ por año y por alumno de escuela primaria en materiales de instrucción, frente a 92 US\$ de los países industrializados ${ }^{5}$. El impacto de esta limitación es aún más grave en momentos de intenso cambio científico-técnico, donde el simple estancamiento implica retroceder. Dos aspectos agravan la seriedad de este problema que estamos comentando. Por un lado, la ausencia de posibilidades de aplicar políticas de mejoramiento de la calidad en momentos de tan intenso cambio científico-tecnológico como el actual implica aumentar sustancialmente el grado de obsolescencia, de aislamiento, de distancia entre los aprendizajes que tienen lugar en la escuela y los aprendizajes socialmente significativos imprescindibles para la participación social.

Pero por otro lado, es preciso considerar el efecto que tiene la larga duración de la crisis. Largos períodos de tiempo sin mantenimiento, sin capacitación, sin posibilidades de atraer hacia la profesión docente a los mejores talentos de la sociedad, provoca efectos irreversibles, cuya superación costará —más tarde - inversiones muy superiores de recursos y de tiempo.

v) Por último, es preciso mencionar una de las manifestaciones menos visibles pero más perversas de la crisis por la que atraviesa la región: la obligación de concentrar esfuerzos y energías en los problemas de coyuntura, abandonando las decisiones de mediano y largo plazo, lugar donde se ubican las principales decisiones educativas. Esta conducta afecta tanto a los gobiernos como a las familias y también en este ámbito la larga duración de la crisis provoca efectos cuya superación será muy difícil y costosa.

\section{Tres niveles de análisis}

El somero diagnóstico sobre el impacto de la crisis, presentado en el punto anterior, no agota la descripción de la actual situación educativa. Contrariamente a ciertas visiones simplificadoras que tienden a analizar unidimensionalmente tanto la situación como las

\footnotetext{
${ }^{5}$ B. Fuller, "Primary School quality in the third world", en Comparative Educational Review, vol. 30, No. 4, nov. 1986.
} 
estrategias para enfrentarla, es preciso asumir que estamos frente a una situación de enorme complejidad, en la cual es posible distinguir al menos tres niveles de análisis, que se corresponden con tres ámbitos distintos de la crisis que afecta a las sociedades modernas: i) el nivel político, donde la democratización y la diversificación de las demandas y de los canales de participación, es un aspecto fundamental, En este nivel la crisis del Estado-Providencia, y especialmente de sus estilos de gestión uniformes y masivos es la expresión más elocuente; ii) el nivel económico, donde la crisis, asociada al endeudamiento externo, constituye — por su profundidad y duración- el fenómeno más importante para los países de América Latina y iii) el nivel pedagógico, cuya crisis tiene manifestaciones anteriores a los fenómenos señalados más arriba y que se asocia estrechamente con la crisis de la cultura contemporánea.

La presencia simultánea de estos tres niveles de análisis y de problemas plantea no sólo enormes desafíos teóricos, sino mucho más serias dificultades prácticas para el diseño y aplicación de políticas públicas. Es frecuente encontrar análisis políticoadministrativos que desconocen la especificidad del problema pedagógico o la viabilidad económica de sus propuestas; inversamente, es posible constatar cómo las estrategias económicas fracasan por la falta de mecanismos administrativos apropiados o ciertos postulados pedagógicos aparentemente correctos desde el punto de vista de las teorías del aprendizaje son poco viables cuando se introducen en sistemas educativos masivos, con administraciones complejas y burocráticas. Por esta razón, si bien parece imprescindible distinguir el ámbito específico de problemas al cual nos referimos, es igualmente importante definir sus articulaciones con los restantes niveles. El reconocimiento de esta complejidad es una condición necesaria tanto para una adecuada comprensión de los fenómenos como para su efectiva atención política. La unidireccionalidad de algunos diagnósticos suele estar en la base de propuestas que tienden a resolver todos los problemas con la simple fórmula de menos Estado y más mercado, independientemente del contexto sociopolítico, económico o cultural e independientemente del sector de actividad al cual nos refiramos.

\section{La crisis del Estado-Providencia y la reforma del Estado en los países avanzados}

Una de las características más importantes de la sociedad moderna es su capacidad para incorporar a toda la población en calidad de fuerza de trabajo desde el punto de vista económico y de ciudadano desde el punto de vista político. Esta incorporación plena -a pesar de las desigualdades en la distribución de los beneficios y del carácter corporativo que asume la participación política- constituye el punto de partida de la discusión sobre la reforma del Estado en los países desarrollados. Desde este punto de vista, los países desarrollados se caracterizan por haber superado la frase de constitución de los mercados nacionales y de satisfacción masiva de las necesidades básicas. Ahora, en cambio, los ciudadanos son más autónomos, mantienen relaciones múltiples y diferenciadas con su medio ambiente y demandan una atención personalizada a sus requerimientos y necesidades. La personalización de los servicios estimula demandas por calidad, que se expresan tanto en las actividades vinculadas con el crecimiento económico (capacitación, investigación científica y técnica, comunicaciones, etc.) como en los servicios asistenciales (salud, educación, previsión social, etc.). Esta situación se refuerza por la creciente competencia internacional por conquistar mercados, que obliga a mejorar permanentemente los niveles de calidad de los productos. En síntesis, sea por la creciente competencia internacional o sea por la competencia política interna para satisfacer las demandas sociales, en los países desarrollados existe un consenso general sobre la necesidad de reformar el Estado con el objetivo de lograr mayores niveles de eficiencia. 
En este contexto, los procesos de reforma del Estado asumen el modelo del cliente como el paradigma de las relaciones sociales y la metodología de la estrategia empresaria como paradigma de la gestión política.

La crisis del Estado en América Latina tiene lugar sobre bases muy diferentes a las que se aprecian en los países desarrollados. En América Latina, la conformación de mercados nacionales es un fenómeno reciente y precario. Para los propósitos de este análisis, baste con recordar que el número de personas que viven en condiciones de pobreza creció de 110 millones en 1960 a 119 millones en 1980, aunque en términos relativos se produjo un descenso del $51 \%$ al $33 \%$. A partir de 1980 y como efecto de la crisis, la pobreza creció tanto en términos absolutos como relativos: el número de personas pobres aumentó a 158 millones, lo cual representa el 39\% de la población. Este promedio regional oculta importantes diferencias entre países $\mathrm{y}$, al interior de los países, entre zonas rurales y urbanas. Sin embargo, a pesar de la heterogeneidad, es posible apreciar que más de un tercio de la población tiene una precaria incorporación al mercado y a la fuerza de trabajo ${ }^{6}$

La explicación de estos resultados alude, obviamente, al desempeño del Estado y de las políticas destinadas a satisfacer los requisitos de equidad social. Al respecto, el efecto regresivo que han provocado numerosas políticas públicas vigentes hasta 1980 ha sido frecuentemente comprobado ${ }^{7}$. A dichos efectos, generados por el estilo concentrador $y$ excluyente del proceso de desarrollo vigente hasta 1980, se agregaron los nuevos problemas derivados tanto de la crisis económica asociada al endeudamiento externo como de las nuevas condiciones generadas por la democratización política y el derrumbe de las experiencias autoritarias.

Dicho sintéticamente, la crisis del Estado-Providencia derivada del excesivo financiamiento asistencial se agudizó por la estatización del pago de la deuda externa, asociada a funciones productivas del Estado o al ineficiente manejo de actividades productivas privadas. Dada la larga duración de la crisis, hoy ya es posible apreciar que el Estado-populista ha sido desmantelado sin que por ello los problemas hayan sido superados. El gasto público se ha reducido en forma dramática, en un contexto donde los efectos recesivos de las políticas de ajuste impiden que otros sectores tengan capacidad para asumir la responsabilidad asistencial que garantizaba el Estado. No estamos, en consecuencia, ante procesos de reforma impulsados por la búsqueda de mayor eficiencia y calidad en la prestación de los servicios sino, más dramáticamente, por el abandono de ciertas funciones que nadie está en condiciones de asumir ${ }^{8}$.

La gravedad del problema es mayor aun si se observa que no sólo los servicios privados no absorben la demanda insatisfecha por el servicio público sino que, al contrario, los efectos de la crisis estimulan una mayor demanda de servicios públicos incluso para sectores que ya habían logrado acceso a la cobertura privada y que han perdido capacidad para mantener dicha cobertura.

El contexto de democratización política en el cual tienen lugar estos procesos agrega nuevos elementos. La democracia supone, en definitiva, la posibilidad real de resolver

\footnotetext{
${ }^{6}$ PREALC/OIT. Deuda social; ¿qué es, cuánto es, cómo se paga? Santiago de Chile, 1988.

${ }^{7}$ Naciones Unidas. ¿Se puede superar la pobreza? Realidad y perspectivas en América Latina. Santiago de Chile, Naciones Unidas, 1980.

8 Juan C. Portantiero. "La múltiple transformación del Estado latinoamericano", en Nueva Imagen, No. 104, 1989. Ricardo Carciofi. La desarticulación del pacto fiscal: una interpretación sobre la evolución del sector público argentino en las dos últimas décadas. Buenos Aires, Cepal, 1989. 
satisfactoriamente los conflictos provocados por la existencia de demandas diferenciadas, a través de mecanismos de concertación y de acuerdo basados no sólo en un mínimo respeto por los intereses del otro sino en la posibilidad real de satisfacer las demandas. La severidad de la crisis provoca enormes dificultades para lograr dicha satisfacción y exacerba la pugna por la apropiación de los escasos recursos disponibles. Los estallidos sociales y la perduración de conflictos violentos en varios países de la región son un indicador del grado de tensión social en el cual se desarrollan los procesos de consolidación democrática. Las reformas del Estado orientadas ya sea a transferir la responsabilidad de ciertos servicios asistenciales a unidades locales sin recursos suficientes o directamente a manos privadas sujetas a las reglas del mercado pueden además de no resolver el problema generar fuerte escepticismo y baja credibilidad acerca del valor de las fórmulas de gestión democrática orientadas a lograr mayores niveles de participación.

\section{La crisis global de la educación: el problema de los valores}

El tema de la crisis de la educación ha sido un tema recurrente en la literatura pedagógica al menos de los últimos cincuenta años. Desde esta perspectiva la crisis no se percibe tanto como un problema de cobertura de los servicios sino como una crisis del proceso pedagógico mismo, entendido como proceso de socialización de las nuevas generaciones por parte de los adultos. Para decirlo brevemente y utilizando las expresiones de A. Arendt ${ }^{9}$, esta crisis está asociada a una de las características centrales de la sociedad moderna: la crisis de la autoridad. La profundidad de este fenómeno es tal que ha afectado campos como el educativo, donde la autoridad había sido aceptada como una necesidad natural, producto de la dependencia del niño con respecto a los adultos.

No es este el lugar para un análisis exhaustivo del problema de la autoridad en la sociedad moderna y sus consecuencias sobre la educación. Para los fines de este artículo interesa destacar, sin embargo, que cuando nos referimos al rol del Estado y de los diferentes agentes de la acción educativa, estamos refiriéndonos a distintas formas de articular los mecanismos de socialización de las nuevas generaciones. El control de estos mecanismos y agentes de socialización constituye, en consecuencia, un factor de enorme relevancia política. Las discusiones de fines del siglo XIX entre el Estado y la Iglesia acerca del papel educador de ambos fue, precisamente, una discusión acerca del control de los mecanismos de socialización secundaria. En la sociedad moderna este debate se produce sobre nuevas bases, donde la escuela (y el maestro) han perdido relevancia desde el punto de vista de su rol socializador. Los agentes socializadores se han diversificado y el papel de las empresas, los medios de comunicación de masas, las asociaciones e instituciones de la sociedad civil es cada vez más significativo. En cierta forma, si se compara la situación actual con la vigencia a fines del siglo pasado, podría sostenerse que se ha producido una inversión en los roles: mientras en el pasado la escuela era la portadora de contenidos socializadores dinámicos frente a los agentes tradicionales (la familia y la Iglesia), actualmente la escuela es percibida como un agente que difunde contenidos obsoletos frente al dinamismo de los medios de comunicación y de las instituciones de la sociedad civil. La diferenciación que existe entre estos agentes (que, en el marco de estrategias privatizadoras de la oferta escolar, tiende a trasladarse también a la escuela) constituye una de las razones por las cuales actualmente la pugna por obtener acceso al conocimiento socialmente más valioso se traduce en una lucha por ocupar los lugares donde dicho conocimiento se produce y se distribuye.

\footnotetext{
${ }^{9}$ H. Arendt. La crise de la culture; huit exercises de pensée politique. París, Gallimard, 1989.
} 
En las sociedades capitalistas avanzadas este debate tiene lugar en el marco de una relativamente alta articulación entre los valores vinculados a la producción y los valores vinculados al consumo: la sociedad promueve el consumo en niveles cuya capacidad de producción está en condiciones de absorber. En los países en desarrollo, en cambio, estos problemas asumen un significado diferente. Por un lado, los niveles de articulación valorativa son muy bajos ${ }^{10}$. La difusión de valores de consumo que superan ampliamente la capacidad de producción es una realidad común a los países en desarrollo, y sus efectos destructores sobre los procesos de acumulación son muy fuertes. Por otro lado, los nuevos valores de consumo y las demandas correspondientes se introducen de manera abrupta, sin que los problemas y demandas del pasado hayan sido totalmente superados.

La pérdida de autoridad es, en estos casos, no sólo generacional sino social. Los jóvenes del mundo subdesarrollado perciben que sus maestros no dominan los aspectos más significativos de la cultura contemporánea y que su sociedad como tal, no participa de la creación de dichos aspectos. Los riesgos de alienación y colonialismo cultural se reactualizan y es por ello que - hoy más que nunca- las opciones tienden a radicalizarse: o se produce un proceso de integración al mundo moderno en condiciones de fuerte dependencia cultural o se abren opciones de aislamiento casi total, sólo posibles con fuertes componentes autoritarios.

El análisis de la socialización en torno a dos valores fundamentales para las estrategias de desarrollo de los países de América Latina puede ilustrar esta situación: la austeridad y la solidaridad.

La austeridad ha sido un valor central en los procesos de acumulación capitalista clásicos, tanto en los países occidentales como orientales. Pero ¿cómo socializar a las nuevas generaciones en la austeridad cuando todos los mensajes que se difunden a través de los agentes socializadores incitan al consumo y cuando los procesos inflacionarios y de especulación financiera de corto plazo desalientan conductas austeras? Las pautas de consumo en países de América Latina, con fuertes desequilibrios en la distribución de los ingresos, permite que en los sectores de ingresos medios se difunda un patrón de consumo similar al de sociedades con niveles de ingresos muy superiores. "Los 18 millones de brasileños y los 7 millones de mexicanos cuyo ingreso medio es de 10.000 dólares anuales hacen suyo y difunden un patrón de consumo calcado de las sociedades avanzadas, acomodando para ello la infraestructura física, energética y de comunicaciones. Los 52 millones de brasileños o los 28 millones de mexicanos con un ingreso medio anual por habitante de 350 dólares están excluidos en buena medida de este patrón de consumo, pero no constituyen un impedimento para que el patrón de desarrollo responda a esta aspiración de las elites ${ }^{11}$. En contextos de este tipo, los portadores del mensaje de la austeridad enfrentan la alternativa de parecer obsoletos y, por lo tanto, no creíbles, o imponer su mensaje por la vía autoritaria de cancelar la difusión de mensajes antagónicos.

El segundo ejemplo lo constituye la socialización dentro de valores de solidaridad. Dado el actual contexto de escasez de recursos y de democratización política, donde la concertación de intereses constituye la fórmula de resolución de conflictos más apropiada,

\footnotetext{
${ }^{10}$ Alain Touraine. Actores sociales y sistemas políticos en América Latina. Santiago de Chile, PREALC, 1987.

${ }^{11}$ F. Fajnzylber. Industrialización en América Latina: de la "caja negra" al "casillero vacío Santiago de Chile, Cepal, 1989.
} 
la socialización en valores y actitudes solidarias se convierte en un requisito básico del éxito de estos procesos. Sin embargo, las tentativas de este tipo chocan con la promoción del éxito individual como paradigma de la conducta y la competitividad como motor del éxito. En contextos de este tipo, la difusión de la solidaridad aparece vinculada más a estrategias de supervivencia que a estrategias de crecimiento, con lo cual el valor queda asociado a un conjunto de situaciones negativas o, al menos, no rige en los ámbitos más dinámicos de la sociedad.

El problema de los valores está ocupando actualmente cada vez mayor atención en los debates sobre políticas públicas y estrategias de desarrollo. Varias razones explican este fenómeno. Desde una perspectiva muy general, la importancia de los valores está asociada al mayor reconocimiento de la dimensión cultural en los procesos de desarrollo; pero en un nivel más específico, este tema está asociado a la relevancia creciente del problema ético en los procesos económicos y en la gestión pública. La enorme gravitación económica del narcotráfico, la corrupción como fenómeno social cada vez más generalizado en la administración de políticas públicas y la especulación financiera, han sido variables cuyo impacto en los resultados de las estrategias de desarrollo no puede seguir siendo considerado en forma marginal. Es probable que se deba a Alain Minc el mérito de haber llamado la atención sobre este problema con mayor énfasis ${ }^{12}$. El postulado central de su trabajo es que los "contrapoderes" que tradicionalmente atemperaban la dominación del capital se han debilitado o han desaparecido. Sin el contrapeso del socialismo, ni del poder sindical, ni de la noción de pecado, el mercado no tiene límites a su dinámica; en este contexto, los riesgos de producir desequilibrios que amenacen la propia estabilidad del capitalismo vuelven a ser muy altos. Invertir en la ética, termina diciendo Minc, es la mejor inversión para el futuro. Pero, desde la perspectiva de este artículo, la pregunta es ¿qué gestión para la difusión de valores? ¿Es posible adoptar una política para los valores o éste es un ámbito que debe quedar librado a la espontaneidad de las necesidades sociales? Las experiencias históricas recientes indican que las políticas (entendidas como diseños explícitos para fomentar ciertos valores) están destinadas al fracaso. Sólo aquellos valores que tienen vigencia real en las relaciones sociales dan contenido al proceso de socialización de las nuevas generaciones. El problema radica en definir modelos de gestión de las instituciones escolares que promuevan un doble movimiento: hacia afuera, incentivando la apertura del mundo escolar al medio que lo rodea: el mundo del trabajo, el medio ambiente natural, las instituciones comunitarias, los medios de comunicación de masas. Pero este movimiento hacia afuera no será exitoso sin fortalecer los valores vinculados a la producción de resultados en el proceso específico de enseñanza-aprendizaje: creatividad, capacidad para resolver problemas, para seleccionar información, para participar en las decisiones y para trabajar en equipo.

\section{Gestión estratégica y crisis}

A partir del análisis efectuado hasta aquí, es posible sostener que en las condiciones de los países en desarrollo, particularmente aquellos más afectados por la deuda externa, el papel del Estado se ubica tanto en el origen del problema como en el principio de la solución. Es cierto que fenómenos tales como el manejo ineficiente de los fondos públicos, el carácter regresivo de muchas políticas de asignación de gastos y la desarticulación entre servicios y capacidad financiera, han estado presentes antes del endeudamiento y que, además, actuaron en la propia lógica que llevó a la crisis. Pero las consecuencias de las crisis son de tal naturaleza que provocan una situación social donde

\footnotetext{
${ }^{12}$ Alain Minc. L'argent fou. París, B. Grasset, 1990.

Digitalizado por RED ACADEMICA
} 
nuevamente se genera una altísima demanda de asistencia social pública para resolver ya no sólo los problemas "normales" de las políticas sociales sino un significativo conjunto de problemas urgentes de supervivencia.

Desde este punto de vista, lo peculiar del actual momento es la coexistencia de procesos de diferenciación y procesos de desigualdad social. Desde el ámbito del proceso de diferenciación, se presentan situaciones vinculadas a la presencia de sectores sociales con capacidad para efectuar demandas sofisticadas que requieren una atención personalizada. Este ángulo del problema se incrementa con la internacionalización de los hábitos culturales y de consumo, que afectando sólo a los sectores sociales que tienen capacidad para satisfacerlas sino al conjunto de la población. Pero desde el proceso de aumento de la desigualdad, la crisis genera demandas de sobrevivencia que estimulan acciones masivas para su solución.

La coexistencia de procesos de diferenciación y de desigualdad presenta — desde el punto de vista de las políticas sociales- un riesgo evidente: establecer un funcionamiento dual donde las demandas de los sectores pobres sean atendidas a través de programas masivos y las demandas de los sectores medios y altos a través de acciones personalizadas. En el caso del sector educativo, como también en los programas de salud, este funcionamiento dual se expresa en el carácter público o privado de los programas y sus diferencias en términos de calidad de los resultados ha sido comprobada reiteradamente. Nadie puede suponer, bajo el pretexto de la necesidad de atender necesidades masivas, que la atención personalizada es menos necesaria en los servicios destinados a sectores de bajos recursos que en los servicios para población de altos recursos. En el caso de la educación, por ejemplo, son numerosas y diversas las evidencias acerca de las peculiaridades con las cuales los niños provenientes de familias de bajos recursos enfrentan las exigencias del proceso de aprendizaje.

El desafío de la política social consiste, precisamente, en lograr incorporar a las acciones públicas el dinamismo que caracteriza a la concepción estratégica vigente en los servicios privados. En los servicios privados, este dinamismo se obtiene a través de la competencia y se asume que la pugna por conquistar mercados es una condición necesaria para la existencia del espíritu estratégico ${ }^{13}$. En el caso de la educación, esta competencia sólo se produce parcialmente en algunos segmentos superiores del sistema o entre sectores sociales con "poder de compra". Programas de alfabetización, de educación básica y de capacitación dirigidos a sectores pobres sólo tienen al Estado como agente disponible para responder a las demandas. Si bien existen organismos nogubernamentales y organizaciones voluntarias (partidos políticos, sindicatos, etc.) que actúan en este terreno con un dinamismo creciente, su capacidad para resolver los problemas en forma masiva es muy limitada y necesitan apoyo estatal permanente.

Si bien la "competencia" no puede ser el factor regulador y dinamizador, no cabe duda que es preciso confrontar la actividad educativa con algún factor externo. Desde este punto de vista, es posible postular que el dinamismo sólo puede provenir de la mayor calificación de las demandas. Permitir mayores grados de control de los procesos y de los resultados por parte de los destinatarios (padres, organizaciones de la comunidad, docentes, etc.), e implementar eficientes sistemas de información al público, constituyen elementos centrales para introducir una dinámica diferente en la gestión de políticas

13 Alain Bienaymé. “¿Cómo puede aprovecharse la estrategia empresarial en el planeamiento de la educación?", en Perspectivas, vol. XIX, No. 2, 1989. 
educativas públicas. Pero la aplicación de estos criterios supone modificar la estructura de la administración educacional, pasando cuotas cada vez mayores de actividades al sistema de administración por programas, a través de contratos de servicios, en lugar del sistema regular de administración.

No caben dudas que ya no es posible seguir insistiendo en estrategias homogéneas para alcanzar objetivos de cobertura universal. Lo "mismo para todos" termina siendo "todo para unos pocos". La aplicación de estrategias diferenciadas constituye un criterio necesario para el logro de objetivos democráticos ya que la homogeneidad está en los productos y no en los procesos. Si el problema se ubica en el logro de resultados homogéneos a través de estrategias diferenciadas y el fortalecimiento de las demandas es concebido como un objetivo importante para el logro de mayores niveles de dinamismo y responsabilidad por los resultados, el diseño de eficaces instrumentos de evaluación de resultados y compensación de diferencias constituye un aspecto central del rol de Estado $^{14}$.

Al respecto, es necesario enfatizar que la evaluación de resultados no puede estar desligada de las posibilidades de acción sobre dichos resultados. Dicho en otros términos, lo importante es medir aquello sobre lo cual se puede actuar. Este principio constituye un eje central en la definición de sistemas de informaciones para los sectores sociales en general y para el educativo en particular. Muchos sistemas de información resultan inoperantes no porque midan cosas superfluas en sí mismas sino porque son superfluas desde el punto de vista de las posibilidades de utilización de dicha información.

\section{¿Cuáles pueden ser las estrategias de cambio?}

La discusión sobre estrategias es una discusión basada en características específicas de cada país. En definitiva, el éxito de una estrategia depende de su grado de adecuación a características locales. Lo exitoso en un contexto puede no serlo en otro. Sin embargo, hay tres líneas de acción que han sido reiteradamente mencionadas por su validez general y que pueden servir de base para la definición de las orientaciones de largo plazo en las decisiones educativas.

La primera de ellas se refiere a la necesidad de definir una nueva jerarquía de prioridades en la asignación de recursos. Desde esta perspectiva, es importante garantizar no sólo una cuota significativa de recursos financieros para la educación, sino su estabilidad en el tiempo. Obviamente, la asignación de recursos estables a los programas educativos dependerá del resultado de las políticas macroeconómicas en términos de crecimiento global y de control antiinflacionario. Pero si bien estos factores son necesarios, la experiencia histórica demuestra que no son suficientes. Paradójicamente, asistimos en la última década del siglo $X X$ a los mismos debates que tuvieron lugar a fines del siglo XIX, cuando algunos políticos de la educación postulaban la necesidad de crear mecanismos de asignación de recursos que garantizaran la estabilidad a través de la independencia de situaciones políticas de corto plazo: fondos escolares permanentes, creados en base a porcentajes sobre determinados impuestos, mecanismos de ajuste por incrementos de matrícula, etc. En este sentido, la independencia del financiamiento educativo con respecto a la coyuntura política debería estar acompañada del diseño de mecanismos eficaces de control social del uso de los recursos.

\footnotetext{
${ }^{14}$ Un mayor desarrollo de este tema puede verse en Juan Carlos Tedesco, "El rol del Estado en la educación", en Perspectivas, vol. XIX, No. 4, 1989.
} 
La prioridad a la educación en la asignación de recursos no implica asumir que la escasez dejará de existir. La restricción será una constante en el futuro próximo y una de las exigencias centrales de la administración educativa será, sin duda, la identificación de estrategias de bajo $\operatorname{costo}^{15}$. Al respecto, parece pertinente agregar una observación que resume, en cierta forma, la experiencia adquirida en estos años y consiste en reconocer que la necesidad de identificar estrategias de bajo costo no significa identificar el bajo costo con el objetivo de la estrategia. Esta observación traduce, en lenguaje un poco más sofisticado, la vieja sabiduría popular según la cual lo barato sale caro y resulta particularmente pertinente para la adopción de estrategias basadas en la utilización de tecnologías de información (educación a distancia, enseñanza de la computación, etc.), que requieren de fuertes inversiones iniciales y cuyas economías se aprecian en el mediano y largo plazo.

La segunda línea de acción se refiere a la necesidad de fortalecer la capacidad de adaptación rápida a necesidades cambiantes en materia de recursos humanos. Desde este punto de vista, la orientación central con respecto a la estructura de los sistemas de acción educativa sería la de establecer amplios márgenes de acción para los actores e introducir fórmulas de evaluación que permitan efectuar los ajustes necesarios y permanentes. Si algo es seguro en el futuro es que los cambios serán frecuentes y rápidos. En ese contexto, es fundamental diseñar instrumentos eficaces de evaluación que permitan actuar antes que los problemas se consoliden y resulte imposible resolverlos sin altos costos sociales y financieros. En este sentido, las fórmulas de descentralización constituyen una vía fértil para mayores grados de pertinencia y de responsabilidad por los resultados. Sin embargo, especialmente en contextos sociales tan heterogéneos y desiguales como los de América Latina, es preciso insistir en que los mecanismos de compensación tienen tanta importancia como los de descentralización.

En tercer lugar y estrechamente asociado al punto anterior, son necesarias respuestas más concertadas a las necesidades educativas. Para garantizar la libertad de acción de un mayor número de actores serán necesarios mayores niveles de organización y de información. Desde este punto de vista, es preciso enfatizar la necesidad de fortalecer los sistemas de información al público acerca de la situación educativa. Las estrategias no pueden seguir concentradas exclusivamente en modificar la oferta escolar. Para que la oferta escolar cambie en un sentido pertinente, para que satisfaga reales necesidades sociales, es necesario que existan fuertes y más calificadas demandas por educación.

\section{Un prerrequisito: el acuerdo educativo nacional}

Por último, es preciso referirse a una precondición de los procesos de reforma del Estado, que ha sido definido en algunos trabajos recientes como un nuevo pacto fiscal o, desde un punto de vista más global, un acuerdo básico entre los diferentes sectores sociales, económicos y políticos sobre el cual iniciar los procesos de cambio y desarrollo.

El punto de partida de esta propuesta es doble: por un lado, la conciencia de la gravedad de la crisis y del riesgo de ruptura de las bases mismas de la organización social. Desde esta perspectiva, se advierte la necesidad de colocar ciertos aspectos de la acción social fuera de la pugna cotidiana. Así como la lucha contra la inflación puede ser el objeto de un consenso básico inmediato en materia económica, la atención universal a la infancia en los aspectos sanitarios y educativos puede constituir un punto importante de

\footnotetext{
${ }^{15}$ Ana M. Corvalán (comp.). El financiamiento de la educación en período de austeridad presupuestaria. Santiago de Chile, OREALC/UNESCO, 1990.
} 
los consensos sociales básicos alrededor de los cuales todos los sectores asuman su cuota correspondiente de compromiso. Por otro lado, el acuerdo está estimulado por razones más prácticas de gobernabilidad. La ejecución de políticas sociales —en particular de políticas educativas - demanda períodos de tiempo que superan los períodos gubernamentales. La estabilidad en ciertas políticas parece ser una condición necesaria para obtener resultados. En el pasado, la estabilidad estuvo frecuentemente asociada al autoritarismo $\mathrm{y}$, por lo tanto, a bajos niveles de legitimidad. Se trata, al contrario, de encontrar fórmulas de estabilidad democrática, sólo posibles sobre la base de consensos legítimos desde el punto de vista de sus contenidos y de su proceso de elaboración.

Una rápida mirada a la historia reciente de América Latina muestra una paradoja que mueve a la reflexión final de este artículo. Durante las décadas de crecimiento (19501980) existieron bases materiales para este consenso. Desde el punto de vista político, en cambio, el consenso no era posible. Existían fuerzas que negaban la posibilidad misma del acuerdo y planteaban la guerra como única solución. En la década de los ochenta, en cambio, las bases materiales para el acuerdo están seriamente deterioradas, pero existen condiciones políticas muy propicias para lograrlo. ¿La austeridad y la escasez según un marco más propicio que la abundancia para obtener el consenso? 